\title{
Simultaneous Bidimensional Spectroscopy of Extended Objects Using Optical Fibers: Application to Seyfert Galaxies
}

\author{
S. Arribas, E. Mediavilla, A. García Marín, B. García Lorenzo and J. L. \\ Rasilla \\ Instituto de Astrofísica de Canarias, 38200-La Laguna (Tenerife) \\ SPAIN
}

\begin{abstract}
In this communication we summarize the main characteristics of three optical fiber systems, namely HEXAFLEX, HEXAFLEX-II, and 2D-FIS, developed for performing simultaneous bidimensional spectroscopy of extended objects. They have been used at the $4.2 \mathrm{~m}$ William Herschel (WHT) and the $2.5 \mathrm{~m}$ Nordic Optical Telescope (NOT), which are sited at the Observatorio del Roque de los Muchachos. These systems have been used to observe the circumnuclear regions of several Seyfert galaxies. They show clear advantages over other instruments based on more classical techniques, at least for these complex objects of small angular size on the sky. Some examples will be given to illustrate this.
\end{abstract}

\section{Introduction}

Simultaneous bidimensional (2D) spectroscopy (1D) of extended objects can be performed by means of optical fibers. To this aim bundles of optical fibers are constructed with the following basic layout: one end of the bundle has the fibers distributed on a two-dimensional array, and is connected to the telescope's focal plane; the other end of the bundle has the fibers aligned at the entrance of the spectrograph simulating the slit (pseudoslit). Hence, when the telescope points to an extended object, each fiber transmits the light coming from a region of the object, and a set of spectra (one per fiber/region) is simultaneously recorded, the individual spectra being well separated on the detector. This application of the fibers has not been sufficiently developed, and only a few systems of this type have been built so far (e.g., Gray, et al, 1982; Vanderriest \& Lemonier, 1988; Barden \& Wade, 1988; Arribas, Mediavilla \& Rasilla, 1991).

However, in principle, they should be more suited to certain applications than the classical methods such as long-slit spectroscopy, tiltable filters, or FabryPerots interferometers. All these techniques require a sequential set of exposures, by shifting the position of the telescope (long-slit) or by varying wavelength (tiltable interference filters or Fabry-Perots). This not only implies that a large amount of observing time is generally required, but also (and this is very important) that serious calibration problems can arise from the fact that the information is not recorded simultaneously. On the contrary, by using optical fibers the entire spectral and spatial information is recorded at the same time (same atmospheric and mechanical conditions). In addition, there are no rel- 
evant constraints on the spectral performance, which basically depends on the characteristics of the spectrograph itself.

Despite these basic advantages, the optical fiber systems pose several technical drawbacks. First, as a consequence of the focal radio degradation (FRD) produced by the fibers, faster collimators must be used, making it difficult to obtain high spectral resolution without extra losses of light. Second, the limited number of fibers that can be used nowadays (mainly as a consequence of the detector size), allows a relatively small spatial coverage if good spatial resolution is required. However, when dealing with complex, and small objects for which low/intermediate resolution is required, these systems seem a very good option. Rapid developments in fiber and detector technologies, as well as improvements to the spectrographs specially designed for use with optical fibers are broadening the field of applicability of these systems in astronomy.

Here we summarize the main characteristics of three optical fiber systems based on the principles outlined above. They have been previously described by Arribas et al (1991) and by García Marín et al (1994). Using of these systems, we are developing a program for studying the circumnuclear regions of Seyfert galaxies. Three galaxies have been studied so far: NGC4151 (Mediavilla et al, 1992) NGC 5728 (Arribas \& Mediavilla ,1993), and NGC 3227 (Mediavilla \& Arribas, 1993b). Here, we will just present a few examples in order to illustrate the advantages of this type of system.

\section{The Systems}

a) HEXAFLEX: The main peculiarity of the HEXAFLEX system (Arribas, Mediavilla \& Rasilla, 1991) is that it allows interchanging two 61 fiber bundles with different spatial configurations (spatial coverage and resolution) very easily and quickly. Both bundles are mounted simultaneously at the entrance of the spectrograph. This idea can be applied to a larger number of bundles, giving the possibility of changing the spatial and/or the spectral resolution and also the mode of observation (Arribas et al, 1993).

b) 2D-FIS: This system is fully described in Garcia Marín et al (1994). It was developed as an extension of the multi-object system for ISIS, which was built in collaboration with the Royal Greenwich Observatory. It links the Cassegrain focus of the $4.2 \mathrm{~m}$ William Herschel Telescope with the ISIS spectrograph (Carter et al, 1993). As ISIS consists of two intermediate-dispersion spectrographs ('arms') operative simultaneously (they share the slit), this system permits to combine the advantages of the fiber systems and a wide spectral range. However, the ISIS spectrograph was not designed for use with fibers having a $f / 11$ collimator and a slit of about $50 \mathrm{~mm}$. Thus, some light is lost as a consequence of the FRD produced by the fibers. The bundle has 125 fibers, 95 for collecting the light from the object, and 30 for the sky background. Therefore, 250 spectra (125 for each arm of the spectrograph) are simultaneously recorded. The acquisition is done with the help of a coherent optical bundle, which links the telescope focal plane (from a position which is off-set with respect to the observing bundle) and a TV CCD camera.

c) HEXAFLEX-II: This is a new version of the HEXAFLEX system for the Nordic Optical Telescope (García Marín et al. 1994). As this telescope has 
excellent seeing quality, the bundles were designed to take advantage of this. As in HEXAFLEX, two bundles can be mounted simultaneously on the spectrograph (FLEX). The bundles have the same configuration as the one designed for 2D-ISIS. However, the acquisition is carried out by means of a mechanical switch which allows the three bundles to be alternatively exchanged each time centering one of them on the optical axis of the telescope. When the telescope points to the object the coherent bundle is centered on the field. This allows to center the object properly with the aid of the TV acquisition system. Then using the mechanical switch one of the two observing bundles can be placed at the center of the field.

\section{Advantages of this type of systems}

The basic advantages of this type of system are those derived from the simultaneousness when recording the spatial and spectral information. Here, we shall briefly comment on the advantages when obtaining line intensity maps, and velocity fields.

a) Intensity maps: The intensity flux corresponding to the spectral lines obtained from fiber data are not affected by the filter effects, which appear when observing through filters, direct images of regions with strong velocity fields (Mediavilla \& Arribas, 1993a). When the filter is broad, contamination appears from others emission lines; when the filter is narrow enough to avoid this, in addition to an accurate knowledge of the systemic velocity, the galaxy's velocity field shifts the wavelength position of the emission lines with respect to the filter transmission profile. The images obtained from fiber data could, however, be affected by photometric instabilities among the different fibers, although after the flat-field correction these are estimated to be small.

b) line ratio maps The good spectral coverage of fiber optics spectroscopy allows us to obtain maps from the intensity ratio of different lines (in this case the photometric instabilities produced by the fibers commented above are removed). Thus with the HEXAFLEX-II and the 2D-FIS systems it is possible, for instance, to obtain maps of $\mathrm{H} \alpha / \mathrm{H} \beta$, [OIII]/H $\beta$, etc. Note that the relatively small spectral range obtained with Fabry-Perots renders them unsuitable to derive this.

c) Velocity fields The velocity fields of Seyfert galaxies are oftenly distorted. Warps or radial velocity fields bend the kinematical axis. This makes diffcult to obtain kinematical information (for instance a velocity curve) from onedimensional spectroscopy (long-slit). Moreover, in some cases the optical nucleus and the kinematical center do not coincide, which may produce misscentering of the slit when using the long-slit technique. These problems are avoided with the use optical fibers. When comparing with a set of long-slit spectra shifted in space, the optical fiber systems present an additional advantage to the simultaneousness, i.e. the regularity in the spatial sampling, and those derived from an accurate knowledge of the relative positions of the spectra. 


\section{Some Results}

A brief comment on some of the results obtained recently by means of this technique can also give an idea of its potential. Here we summarize some of the results obtained on NGC5728 and NCG3227.

a) Hidden nucleus in NGC 5728: Arribas \& Mediavilla (1993) suggest that the true nucleus of this galaxy is hidden at the site of the kinematical center. This result is obtained after comparing the $\mathrm{H} \alpha$ intensity map and the velocity field. This comparison can be done with good accuracy as they were obtained from the same set of spectra (simultaneouly recorded), their relative positions being well known. Recent images taken with the Hubble Space Telescope (Wilson et al., 1993) support the existence of the obscured nucleus.

b) Evidence of an offset active nucleus in NGC 3227: In this galaxy Mediavilla \& Arribas (1993b) found an offset between the position of the BLR and the kinematical center. It was possible to obtain this result once the accurate position of the BLR were fixed over the velocity field. In addition, the existence of double peaked lines was also found, indicating the presence of two different kinematical systems, one associated to the active nucleus. The analysis of the line ratios corresponding to each component indicate that they are originated under different physical conditions.

Acknowledgments. We are grateful to D.Carter,and D. Gellatly, from the RGO, and to B. Petersson (Uppsala) for their colaboration. We also thank to ORM staff for their help at the observatory.

\section{References}

S. Arribas, E. Mediavilla \& J. L. Rasilla, 1991, ApJ, 369; p. 260.

S. Arribas \& E. Mediavilla, 1993, ApJ, 410; p. 552.

S. Arribas, E. Mediavilla \& J. L. Rasilla, 1993, in Fiber Optics in Astronomy II (ASP Conf. Ser. 37), ed. P.M. Gray, p. 322.

S. C. Barden \& R. C. Wade,1988, in Fiber Optics in Astronomy I (ASP Conf. Ser. 3), ed. S. C. Barden, p. 113.

D. Carter, C. R. Benn, R. G. M. Rutten, J. M. Breare, P. J. Rudd, D. L. King, R. E. S. Clegg, V. S. Dhillon, S. Arribas, J.L. Rasilla, A. Garca, C. R. Jenkins \& P. A. Charles, 1993, WHT - ISIS Users' Manual, Isaac Newton Group, La Palma.

A. García Marín, J.L. Rasilla, S. Arribas \& E. Mediavilla 1994, to appear in "Instrumentation in Astronomy", SPIE vol. 2198.

P. M. Gray, M. M. Phillips, A. J. Turtle \& R. Ellis, 1982, Proc. ASA 4 (4), p. 477.

E. Mediavilla, S. Arribas \& J. L. Rasilla, 1992, ApJ, 396; p. 517.

E. Mediavilla \& S. Arribas, 1993a, Nature, vol. 365, p. 420.

E. Mediavilla \& S. Arribas, 1993b, Fiber Optics in Astronomy II (ASP Conf. Ser. 37), ed. P.M. Gray, p. 331.

C. Vanderriest \& J.-P. Lemonnier, 1988, Instrumentation for Ground-Based Optical Astronomy, ed. L. B. Robinson, p. 304. 